\title{
Surgical treatment of degenerative and traumatic spinal diseases with expandable screws in patients with osteoporosis: 2-year follow-up clinical study
}

\author{
Roberto Gazzeri, MD, Raffaelino Roperto, MD, and Claudio Fiore, MD \\ Department of Neurosurgery, San Giovanni Addolorata Hospital, Rome, Italy
}

OBJECTIVE Pedicle screw instrumentation of the osteoporotic spine carries an increased risk of screw loosening, pullout, and fixation failure. A variety of techniques have been used clinically to improve pedicle screw fixation in the presence of compromised bone. Pedicle screws may be augmented with cement, but this may lead to cement leakage and result in disastrous consequences. To avoid these complications, a multiaxial expandable pedicle screw has been developed. This was a prospective, single-center study designed to evaluate the clinical results of patients with osteoporosis with traumatic and degenerative spinal diseases treated with expandable pedicle screws.

METHODS Thirty-three patients (mean age 61.4 years) with osteoporosis and traumatic or degenerative spinal diseases underwent spinal posterior fixation with expandable screws. Preoperative and postoperative visual analog scale (VAS) for pain and Oswestry Disability Index (ODI) questionnaire scores were obtained. The immediate postoperative screw position was measured and compared with the final position on lateral plain radiographs and axial CT scans at the 1- and 2-year follow-up examinations.

RESULTS A total of 182 pedicle screws were used, including 174 expandable and 8 regular screws. The mean preoperative patient VAS score improved from 8.2 to 3.6 after surgery. The mean ODI score improved from $83.7 \%$ before surgery to $29.7 \%$ after the operation and to $36.1 \%$ at the final follow-up. No screw migration had occurred at the 1 -year follow-up, but 1 screw breakage/migration was visualized on spinal radiography at the 2-year follow-up.

CONCLUSIONS The results of this study show that the multiaxial expandable pedicle screw is a safe and practical technique for patients with osteoporosis and various spinal diseases and adds a valuable tool to the armamentarium of spinal instrumentation.

http://thejns.org/doi/abs/10.3171/2016.3.SPINE151294

KEY WORDS lumbar osteoporosis; vertebral fracture; spinal fixation; expandable pedicle screw; spinal fracture; listhesis; OsseoScrew; degenerative spinal disease; osteopenic vertebra; technique

$\mathrm{O}$ STEOPOROSIS is a major global health problem, with more than 10 million people currently diagnosed with this ailment., ${ }^{2,14}$ Although $80 \%$ of patients with osteoporosis are women, a considerable number of men are also affected. Due to increasing life expectancy, the number of elderly patients with osteoporosis affected by spinal diseases will continue to grow. Osteoporosis reduces bone quality through negative bone remodeling, predisposing patients to spinal fracture, deformity, and stenosis; thus, surgical correction of these problems in such patients is difficult. Although pedicle screws are the most commonly used device to achieve posterior fixation in the thoracic and lumbar spine, their use is relatively contrain- dicated in patients with osteoporosis because of the risk of failure at the bone-screw interface, leading to screw pullout, loosening, and migration. ${ }^{11,19,23}$ Major complications include pseudarthrosis, and hardware failure secondary to poor fixation in osteoporotic bone. Obtaining adequate screw purchase remains a challenge in poor bone quality. As a result, a variety of methods have been developed to improve pedicle screw fixation in the osteoporotic spine. These include the use of bigger and longer screws, optimizing the pedicle fit of the screw, undertapping the screw trajectory, and selecting the optimal screw trajectory. 1,30

Nevertheless, implant failure still occurs with increasing frequency. To enforce the construct anchorage in weak

ABBREVIATIONS BMD = bone mineral density; $\mathrm{CaP}=$ calcium phosphate; $\mathrm{DEXA}=$ dual-energy $\mathrm{x}$-ray absorptiometry; ODI = Oswestry Disability Index; PMMA = polymethylmethacrylate; VAS = visual analog scale.

SUBMITTED October 30, 2015. ACCEPTED March 7, 2016.

INCLUDE WHEN CITING Published online June 17, 2016; DOI: 10.3171/2016.3.SPINE151294. 
osteoporotic vertebrae, several authors have reported augmenting conventional pedicle screws with polymethylmethacrylate (PMMA) - and calcium phosphate (CaP)based cements. ${ }^{3,4,24}$ However, the drawbacks of cementaugmented screws, either cannulated or inserted using the kyphoplasty or vertebroplasty technique, are the significant potential leakage and the risk of pulmonary embolism. ${ }^{13,26}$

The development of alternative pedicle screw designs to increase the pullout strength of the screw-bone interface was prompted by recognition of potential fixation complications. A new solution for increasing pedicle screw fixation has been suggested by several investigators who developed expandable pedicle screws. These screws have been shown to result in a $30 \%$ increase in bone pullout strength compared with conventional pedicle screws, and a $50 \%$ increase in pullout strength in osteoporotic bone. ${ }^{33}$ In this study, we report the radiological and clinical results of pedicle screw fixation in patients with osteoporosis and degenerative and traumatic spinal diseases and evaluated whether fixation of expandable screws is a safe, useful surgical technique for the treatment of these conditions.

\section{Methods}

\section{Implant Description}

The OsseoScrew Spinal Fixation System (Alphatec Spine) is a new-generation expandable pedicle screw fixation system for use in degenerative and traumatic thoracolumbar spine disease in combination with rod stabilization. ${ }^{10,33}$ The pedicle screw OsseoScrew is an expansion screw that has Grade 5 titanium in its inner portion that guarantees the seal of the screw and prevents its breakage, and of commercially pure titanium (Grade 2, which ensures elasticity) in the outer part of the screw (Fig. 1). The internal shaft is $3.6 \mathrm{~mm}$ in diameter in the $6.5-\mathrm{mm}$ screw and 4.3 $\mathrm{mm}$ in diameter in the 7.5-mm screw. The inner diameter of the screw determines the fatigue strength while the outer diameter of the screw determines the pullout strength.

The outer part of the screw of commercially pure titanium can be expanded in its central portion and always at $26.85 \mathrm{~mm}$ from the head of the screw. This expansion is manually controllable by the operator with the aid of intraoperative radiographs. The $6.5-\mathrm{mm}$ screw opens up to a maximum diameter of $10 \mathrm{~mm}$, while the $7.5-\mathrm{mm}$ screw expands to a maximum diameter of $11 \mathrm{~mm}$ just past the opening of the pedicle-vertebral body junction.

After anatomical preparation for insertion of the pedicle screw is achieved using a classic surgical technique, a placement probe is used to visualize the location of the center of the expansion area of the screw. The probe contains cutting flutes to ensure the ball of the probe (and thus the expansion of the screw) is anterior to and not within the pedicle.

After insertion of the screw in the vertebral body, the expansion of the screw occurs thanks to a manual mechanism triggered by the operator, which has the terminal part that is screwed into the distal region of the screw. The front of the screw is then pulled posteriorly to expand the commercially pure titanium skin over the titanium alloy inner component. Under fluoroscopic view, the operator turns a handle on the screwdriver in a clockwise direction to expand the screw in the vertebral body. Furthermore,

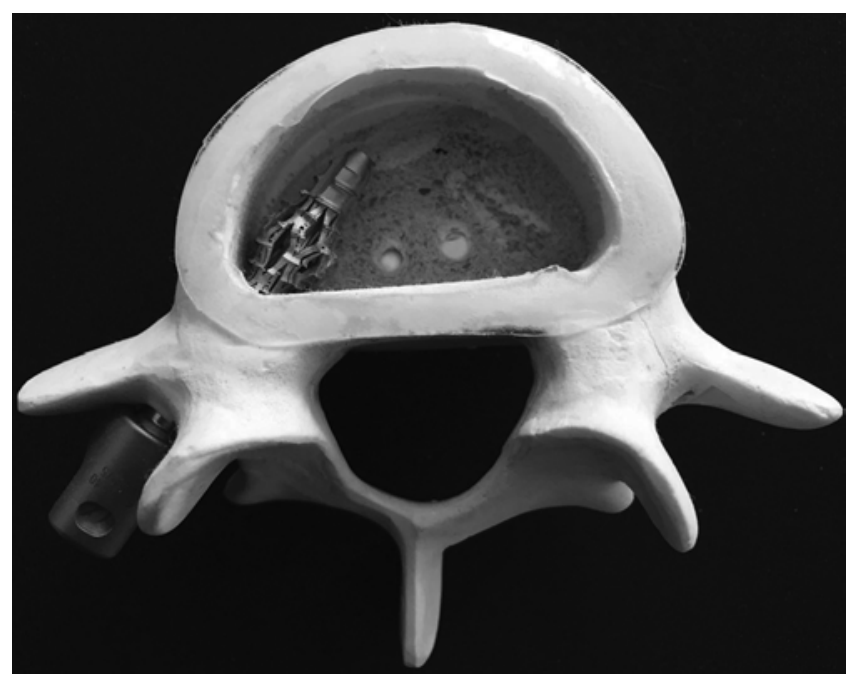

FIG. 1. Photograph of a model of a lumbar vertebra with the OsseoScrew pedicle screw inserted in the pedicle and expanded inside the vertebral body.

the screw is perforated to allow cement injection. The commonly used diameters of the screw are $6.5 \mathrm{~mm}$ and $7.5 \mathrm{~mm}$. In case of malposition of the screw, the expansion of the screw may be collapsed or undeployed during surgery or subsequent revision surgeries with a separate removal instrument.

\section{Patient Population and Data Collection}

A consecutive prospective series of 33 patients with osteoporosis between May 2010 and December 2012 underwent surgery with posterior spinal fixation with expandable pedicle screws. The preoperative pathology of patients included osteoporotic compression/burst vertebral fracture, degenerative spondylolisthesis, and spinal canal and/or foraminal stenosis. Inclusion criteria were: 1) patients over 18 years of age; 2) demonstration by dual-energy x-ray absorptiometry (DEXA) bone mineral density (BMD) examination of deficiencies ranging from osteopenia to severe osteoporosis, with a T-score of less than -1.0 according to the WHO criteria; 3 ) no response to nonoperative treatments; and 4) evidence of spinal trauma, or degenerative or deformative spinal disorders requiring stabilization of the thoracolumbar and lumbar spine.

Patients were excluded from this study in cases of 1) active or previous history of spinal infection; 2) life expectancy less than 2 years; 3 ) severely increased risk for surgery due to cardiovascular or pulmonary diseases, or other significant comorbidities; 4) spondylolisthesis > Grade III; and 5) allergies to any of the device components.

\section{Outcome Measurements}

The radiological results of the patients were evaluated. All patients were examined using spinal CT or MRI to define the surgical indication and to measure the pedicle diameter and length prior to surgery. Spinal radiography and CT scans were performed immediately after surgery, at 6 months postoperatively, and then at 1 and 2 years.

Postoperative radiological analysis was performed us- 
TABLE 1. Clinical data of patients undergoing multiaxial expandable pedicle screw fixation

\begin{tabular}{|c|c|}
\hline Variable & Value (\%) \\
\hline No. of patients & 33 \\
\hline Sex (male/female) & $7 / 26$ \\
\hline \multicolumn{2}{|l|}{ Diagnosis } \\
\hline Burst/compression fracture & $16(48.5)$ \\
\hline Spinal stenosis & $7(21.2)$ \\
\hline Spinal listhesis & $7(21.2)$ \\
\hline Failed back surgery syndrome & $3(9)$ \\
\hline \multicolumn{2}{|l|}{ Location of the lesion } \\
\hline Lumbar spine & $12(36.6)$ \\
\hline Lumbosacral spine & $10(30.2)$ \\
\hline Thoracolumbar spine & $9(27.2)$ \\
\hline Thoracic spine & $2(6)$ \\
\hline \multicolumn{2}{|l|}{ Surgical indications } \\
\hline Painful osteoporotic fracture & $14(42.4)$ \\
\hline Degenerative spinal diseases & $17(51.5)$ \\
\hline Persistent back pain after vertebroplasty & $2(6)$ \\
\hline \multicolumn{2}{|l|}{ Cause of injury in traumatic vertebrae } \\
\hline Fall & 10 \\
\hline Lifting of heavy objects & 3 \\
\hline Undetermined causes & 2 \\
\hline Standing-up motion & 1 \\
\hline \multicolumn{2}{|l|}{ Comorbidities } \\
\hline Diabetes mellitus & 6 \\
\hline Rheumatoid arthritis & 4 \\
\hline Asthma & 3 \\
\hline Thyroid tumor & 2 \\
\hline Benign prostate hypertrophy & 2 \\
\hline Hypertension & 14 \\
\hline
\end{tabular}

ing computer software (Syngo, Siemens). The immediate postoperative screw position was measured and compared with the final position evaluated on a lateral plain radiograph and axial CT scan. The distance from the screw tip of each level to the anterior cortex of the vertebral body was measured on the CT scan.

The distance from the deepest screw tip of each level to the anterior cortex of the vertebral body and to the upper endplate was measured and recorded on the lateral radiograph. Preoperative, postoperative, and final follow-up kyphotic angles were measured between the upper endplate line of most upper-instrumented vertebrae and that of the lowest instrumented vertebral body. Fusion was considered successful when there was no sign of radiolucency around the pedicle screws and the dynamic radiographs showed no movement between the fixed segments. Complications related to the hardware (screw loosening, migration, or rupture) were also evaluated based on clinical and radiographic data.

Regarding clinical symptoms, an independent researcher interviewed all patients. All questionnaires were supplemented by a review of the patient's medical records. Preoperative and postoperative pain was measured using a visual analog scale (VAS, 0-10), and pain and functional capacity was assessed with the Oswestry Disability Index (ODI) questionnaire. The values obtained preoperatively, immediately after surgery, and 1 and 2 years after surgery were compared.

\section{Results}

\section{Patient Demographics}

A total of 33 patients were enrolled in this study. The subjects included 7 men and 26 women. The mean age was 61.4 years (range 39-78 years), with a mean followup of 35.2 months (range 24-55 months). Spinal disease on admission was osteoporotic compression/burst fracture in 16 patients $(48.5 \%)$, and degenerative disease in $17 \mathrm{pa}-$ tients $(51.5 \%)$. The cause of injury in traumatic vertebrae included a fall in 10 patients, lifting of heavy objects in 3 patients, a standing-up motion in 1 patient, and undetermined causes in 2 patients.

The location of the lesion was the lumbar spine in 12 patients, lumbosacral in 10 patients, thoracic in 2 patients, and thoracolumbar junction in 9 patients. The constructs were 2-segment fusion in 13 cases, but there were also 14 cases of 3-level and 6 cases of 4-level segment fusion. Demographic characteristics of the included patients, their clinical data, and the location of the lesions are summarized in Table 1. Osteoporosis was classified as senile or postmenopausal in 25 patients, and the remaining 8 patients had complications of rheumatoid arthritis, thyroid tumor, or were receiving steroid therapy. DEXA BMD examination performed preoperatively showed a mean $\mathrm{T}$ score of -2.8 ( -1.8 to 4.0$)$. The cutoff T-score was 1.8 . In 27 patients, the T-score was lower than -2.5 (osteoporotic), 6 patients had osteopenia (between -1.8 and -2.5 ), and in all cases DEXA was recorded from the spine. All patients with osteopenia were receiving calcium and vitamin D supplements preoperatively. The mean body mass index was $25.8 \mathrm{~kg} / \mathrm{m}^{2}$ (range $18.3-34.3 \mathrm{~kg} / \mathrm{m}^{2}$ ). Comorbidity factors were found in 21 of 33 patients.

A total of 182 pedicle screws were used, including 174 expandable and 8 standard screws. The 8 short standard screws were placed as supplementary fixation in fractured vertebrae or in a vertebral body that had previous vertebroplasty. In 1 case, an expandable screw was augmented with cement (Table 2). In the middle of the constructs, 32 expandable screws and 8 regular screws were placed. In 1 patient in whom a previous kyphoplasty/vertebroplasty had been performed, we used shorter regular screws in these vertebrae and expandable screws in the upper and lower vertebrae.

The mean operative duration was $180 \pm 66.3$ minutes (range 75-260 minutes), and the mean estimated blood loss was $240 \pm 85 \mathrm{ml}$ (range $50-450 \mathrm{ml}$ ). The minimal follow-up period was 24 months for all patients.

\section{Clinical Outcomes}

The mean ODI score improved from $83.7 \%$ (range 65\%$97 \%$ ) before surgery to $29.7 \%$ (range $0 \%-42 \%$ ) after surgery at 6-month follow-up. Functional capacity and pain evaluated by the ODI showed significant improvement compared with that between preoperative and postoperative periods, 
TABLE 2. Lesion level and screw number data

\begin{tabular}{cccc}
\hline Level & Level of Lesion & Total No. of Screws & Expandable Screws \\
\hline T-8 & 0 & 2 & 2 \\
\hline T-9 & 1 & 0 & 0 \\
\hline T-10 & 0 & 6 & 6 \\
\hline T-11 & 1 & 14 & 12 \\
\hline T-12 & 5 & 16 & 14 \\
\hline L-1 & 5 & 12 & 8 \\
\hline L-2 & 2 & 14 & 14 \\
\hline L-3 & 5 & 32 & 32 \\
\hline L-4 & 14 & 30 & 30 \\
\hline L-5 & 13 & 36 & 36 \\
\hline S-1 & 8 & 20 & 20 \\
\hline
\end{tabular}

including the 12- and 24-month follow-up (Fig. 2). The mean preoperative VAS score of 8.2 (range 6-10) improved to 3.66 (range 2-4) after surgery. Based on the VAS score for back pain, pain intensity was improved at discharge, at 6 months, and at the 12- and 24-month follow-up evaluations (Fig. 3).

Clinical success was defined as $>25 \%$ improvement rate in ODI score, $>2$-point improvement in VAS score, $>$ $50 \%$ patient subjective recovery rate, and no major complications related to the device, as suggested by Shim et al. ${ }^{29}$ According to these criteria, clinical success at 2-year follow-up was observed in 31 (93.9\%) of 33 patients.

\section{Radiological Outcomes}

Spine radiographs and CT scans were obtained postoperatively, and at 6,12 , and 24 months postoperatively in all patients except in 7 in whom the 6-month CT scan was not performed. There was no appreciable screw loosening or migration on CT scans at 6- and 12-month follow-up.

At 2-year follow-up, there was no sign of radiolucency around the pedicle screws on plain radiographs and spinal CT scans, except for 1 case of screw breakage and migration (outer expandable part of the screw) in which radiolucency was present around the broken screw and around the other screw inserted into the same vertebral body. On spinal radiographs, the average immediate postoperative horizontal and vertical screw distance from the screw tip

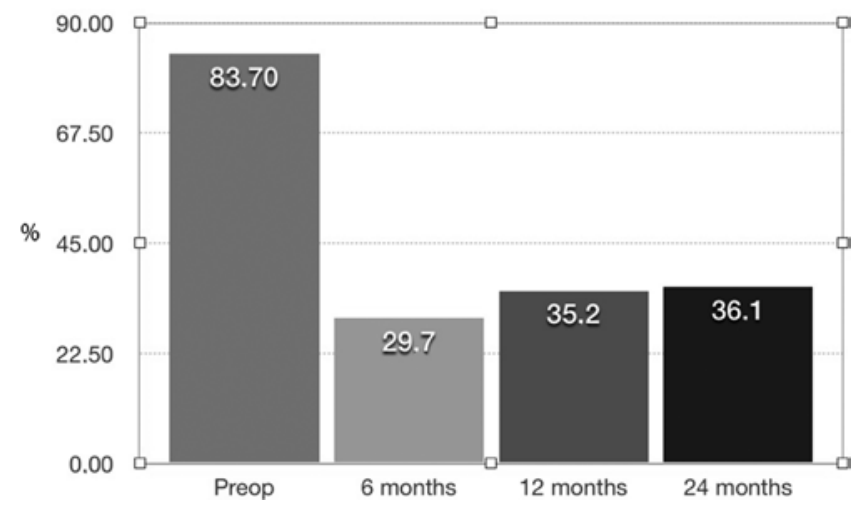

FIG. 2. Graph of patient functional capacity and pain evaluated by ODI scores. Mean scores are given within the bars. to the anterior cortex and upper endplate of the vertebra was $11.3 \mathrm{~mm}$ and $9.9 \mathrm{~mm}$, respectively, and at the final follow-up, these distances were $12.2 \mathrm{~mm}$ and $11.3 \mathrm{~mm}$, respectively (Fig. 4). On CT scans, the average postoperative distance from the screw tip of each level to the anterior cortex of the vertebral body was $11.2 \mathrm{~mm}$ (range $6.5-14.8 \mathrm{~mm}$ ), and the distance at final follow-up was 11.6 $\mathrm{mm}$ (range 6.6-15 mm; Fig. 5) The kyphotic angle was significantly decreased after surgery in all patients treated for vertebral fracture. The kyphotic angle was improved from an average of $19.9^{\circ}$ (range $3.5^{\circ}-48.2^{\circ}$ ) to $10.4^{\circ}$ (range $3.1^{\circ}-29.2^{\circ}$ ). The changes in the kyphotic angle over time did not differ significantly postoperatively, with a loss of kyphosis correction of $2.8^{\circ}$ at final follow-up (Table 3).

\section{Complications After Surgery}

There were no cases of severe morbidity after surgery (no deaths, no myocardial infarctions, and no pulmonary emboli). In 3 patients, an adverse event occurred in the perioperative period. In a case of failed back surgery syndrome and another case of spinal lumbar stenosis, a few days after laminectomy and spinal fixation a subcutaneous CSF leak developed. A spinal CT scan showed no relation between the pedicle screws and the dural leak. A lumbar drain was inserted for 3 days and the CSF leak was resolved expeditiously in 1 patient, while the other patient needed a new surgical approach to repair the dura. In 1 case of screw misplacement detected at the postoperative CT scan, the misplaced screw was removed and replaced a few days after surgery.

Expandable screw breakage with anterior migration of the outer part of the screw occurred in 1 patient $(3 \%)$ at 24 months after surgery. The pedicle screw was removed because of local discomfort and the construct was reinforced cranially and caudally. In 1 case of spinal stenosis with acute worsening of back pain at 1-year follow-up, expandable screws were used to reinforce the construct by gaining additional fixation in the caudal vertebral segment, achieving improvement in pain.

\section{Discussion}

The number of elderly patients requiring thoracolumbar and lumbar fusion is rapidly growing as life expectancy increases. However, the stability of the fixation depends

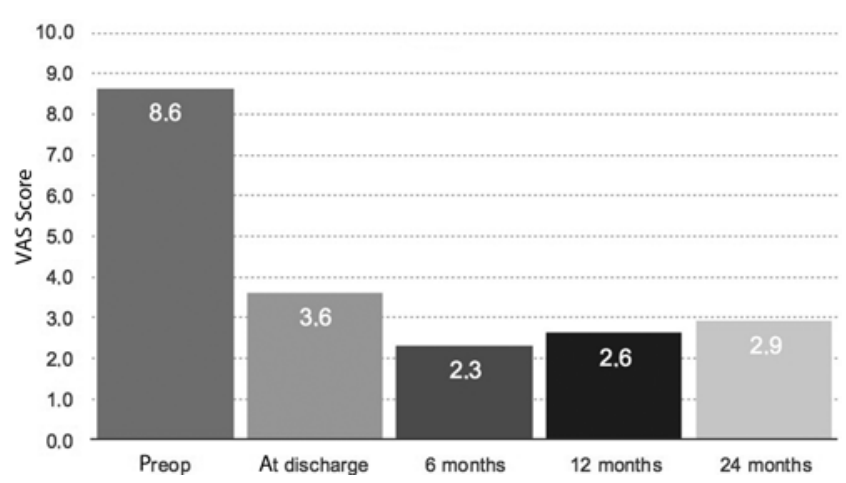

FIG. 3. Graph illustrating patient pain evaluated by VAS scores. Mean scores are given within the bars. 


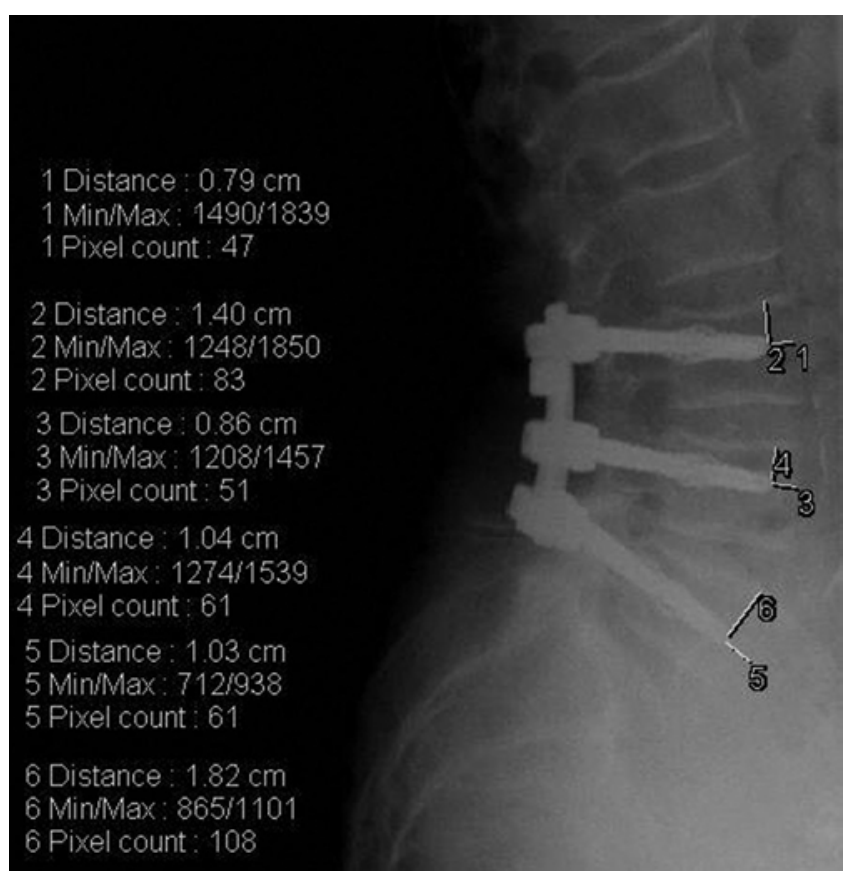

FIG. 4. Lateral spine radiograph showing measurements of the distance from the deepest screw tip of each level to the anterior cortex of the vertebral body and to the upper endplate, to analyze screw migration.

on the purchase of the screws in the pedicle and in the vertebral body. ${ }^{5,23}$ Although pedicle screw instrumentation has become extremely popular over the last decade yielding favorable clinical and radiological outcomes for such patients, major concerns are osteoporosis-related problems such as screw pullout, screw loosening, and fusion failure. ${ }^{5}$ Because the pullout strength of regular screws is decreased by the loosened quality and quantity of the vertebral cancellous bone, fixation failure is likely to happen in elderly patients with osteoporosis.

The pullout and loosening of pedicle screws, resulting from a failure of screw fixation, remains a significant clinical problem, especially in patients with poor bone quality because it may lead to loss of the sagittal alignment or nonunion, with more frequent screw movements within the vertebra than within the normal spine, leading to a failure rate of $0.6 \%-11 \% .^{11,19,23} \mathrm{~A}$ variety of techniques have been used clinically to improve pedicle screw fixation in the presence of compromised bone. Many experimental studies have proved that the stiffness and strength of pedicle screw fixation can be increased when the pedicle screw is augmented with various cements. ${ }^{9}, 16,17,21,24,40$ The optimum augmentation method of pedicle screw fixation strength continues to be debated. Cement-augmented screws are reported to offer a higher resistance to pullout forces than standard screws, with the average pullout resistance ranging from $150 \%$ to $250 \%$ of that of standard screws. $3,18,24,26,40$ But cement leakage is the most common and troublesome complication in posterior cement augmentation with pedicle screw fixation, either cannulated or inserted using the vertebroplasty technique, with a rate of up to $26.2 \% .4,22,27$ Injection of cement into the vertebral body during its liquid phase presents the potential for leakage, increasing the risk of neural injury. Additionally, instantaneous risk
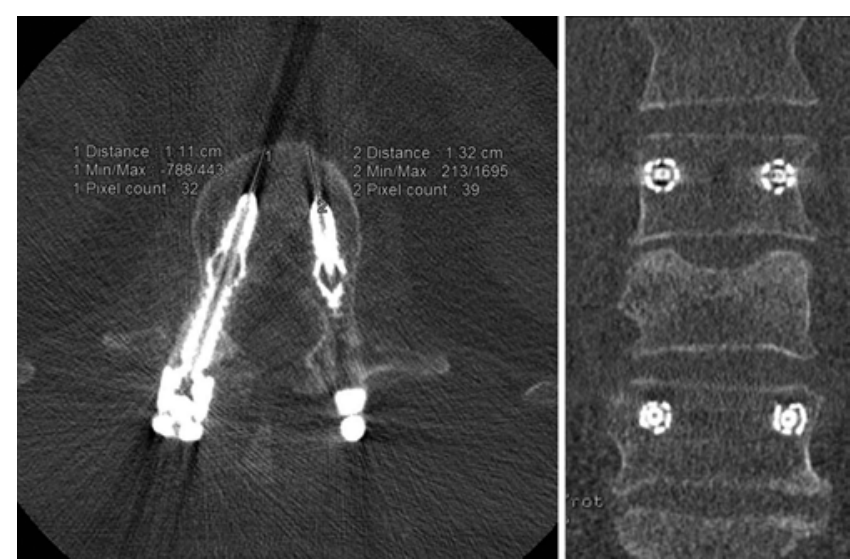

FIG. 5. Postoperative CT scans. Left: The distance from the screw tip of each level to the anterior cortex of the vertebral body was measured on an axial CT scan to analyze screw migration/pullout. Right: The coronal view shows the expansion of the outer part (commercially pure titanium) of the screws in the vertebral body.

resulting from cement leakage into the spinal canal is the result of the high exothermic polymerization temperature reaction of PMMA and the risk of pulmonary embolism. However, long-term risks are secondary to the presence of cement compression in the spinal canal, the increased risk for adjacent-segment fractures, and the possibility of providing a host for an infection. Furthermore, long-term exposure to PMMA can result in its toxic monomer methylmethacrylate being absorbed into the blood, resulting in embolic events and hypotension. An alternative bone cement is $\mathrm{CaP}$ cement, a biodegradable material that gradually degrades allowing newly formed trabeculae to take its place. ${ }^{32,39}$ There is no heat produced and thus no risk of thermal necrosis of the surrounding tissue, but the potential risk of leakage still exists. Compared with PMMA, $\mathrm{CaP}$ cement has a weaker pullout force, although a rate of increase in pullout strength of $244 \%$ for CaP augmented screws has been reported compared with standard screws in the osteoporotic spine. ${ }^{24,31}$

Another drawback is the revision of cement-augmented screws, with a difficult removal due to the rigid interdigitations of cement remnants attached to the pedicle screw, resulting in large vertebral body defects with limited backup strategies. Therefore, fixation in the severely osteoporotic spine represents a challenge, regardless of technique. To avoid these complications, the expandable screw was developed. ${ }^{36,37}$

TABLE 3. Radiographic parameters to analyze screw migration

\begin{tabular}{|c|c|c|c|}
\hline Parameter & Preop & Postop & $\begin{array}{c}\text { 24-Mo } \\
\text { Follow-Up }\end{array}$ \\
\hline $\begin{array}{l}\text { Distance of screw from anterior } \\
\text { vertebral body margin (CT scan) }\end{array}$ & & $11.2 \mathrm{~mm}$ & $11.6 \mathrm{~mm}$ \\
\hline $\begin{array}{l}\text { Horizontal screw distance (radio- } \\
\text { graph) }\end{array}$ & & $11.3 \mathrm{~mm}$ & $12.2 \mathrm{~mm}$ \\
\hline Vertical screw distance (radiograph) & & $9.9 \mathrm{~mm}$ & $11.3 \mathrm{~mm}$ \\
\hline $\begin{array}{l}\text { Kyphotic angle (in burst/compres- } \\
\text { sion fracture) }\end{array}$ & $19.9^{\circ}$ & $10.4^{\circ}$ & $13.2^{\circ}$ \\
\hline
\end{tabular}


The fixation strength of the expanded screws is greater than that of the standard screw. Cook et al. evaluated the biomechanical testing of an expansion screw with a fullshaft expansion mechanism. The pullout resistance increased by $20 \%$ compared with the standard screw (313 vs $408 \mathrm{~N}$ ), and in the low BMD group, the expansion screw increased the pullout resistance by $50 \%$ compared with the standard screw. ${ }^{7}$ The impact of a small-sized distal expansion mechanism added to a standard pedicle screw was recently analyzed. The slight enlargement of the distal screw shaft diameter by the expansion mechanism caused an increase of about $20 \%$ of pullout resistance compared with standard screws. ${ }^{15}$

In another study, Wan et al. reported on the pullout testing of midshaft expansion pedicle and standard screws 6 months after insertion in an osteoporotic calf vertebrae, with a $60 \%$ increase in the failure load (596 vs $951 \mathrm{~N}$ ) provided by the expansion screw. ${ }^{34}$ Vishnubhotla and colleagues evaluated the mechanical properties of the OsseoScrew multiaxial expandable screw. ${ }^{33}$ In particular, with the expansion mechanism located between the distal two-thirds of the screw and radial expansion to $10 \mathrm{~mm}$, a net increase of pullout load by $30 \%$ was achieved. Energy required to cause the ultimate failure of the bone-implant interface was about $160 \%$ greater for the expansion compared with the standard screw.

From a purely mechanical perspective, the allowable load placed on a screw is dependent on the amount of the surrounding material that contacts the thread. ${ }^{20}$ The significant increase in pullout strength results from the increased contact area between the expanded pedicle screw and the surrounding bone, achieving a greater distribution of forces. Increased fixation strength results from the compaction of the bone surrounding the expansion of the screw. The trabecular bone surrounding the expandable portions of the multiaxial expandable screw is compressed and becomes denser than it was before screw expansion. This interaction between the screw and the trabecular bone increases the fixation strength at the screw-bone interface. The expansion of the screw also improves the fixation strength by allowing greater bone contact in the vertebral body ventrally to the pedicle insertion, without an increase in screw length or diameter inside the pedicle. Furthermore, the geometry and location of the expanded portion of the screw is extremely beneficial, because roughly $60 \%$ of the pullout strength of a pedicle screw is dependent on the cortical bone of the pedicle itself, while only $15 \%-20 \%$ depends on trabecular bone of the vertebral body. 12,35

The OsseoScrew has an expandable region that locks the screw into the neck of the pedicle, in a manner that is similar to how a molly bolt is used in dry wall. This expansion zone is designed to optimize pedicle fixation. ${ }^{33}$ Histologically, newly formed bone tissue may grow inside the expanded area of the screw. This may improve the pullout strength of the expandable screw and lead to greater fixation despite the poor bone quality of the osteoporotic vertebra.

Previous studies reported that expandable screws of different shapes were removed in $1.8 \%-4 \%$ of patients because of pseudarthrosis and local discomfort. ${ }^{28}$ In all

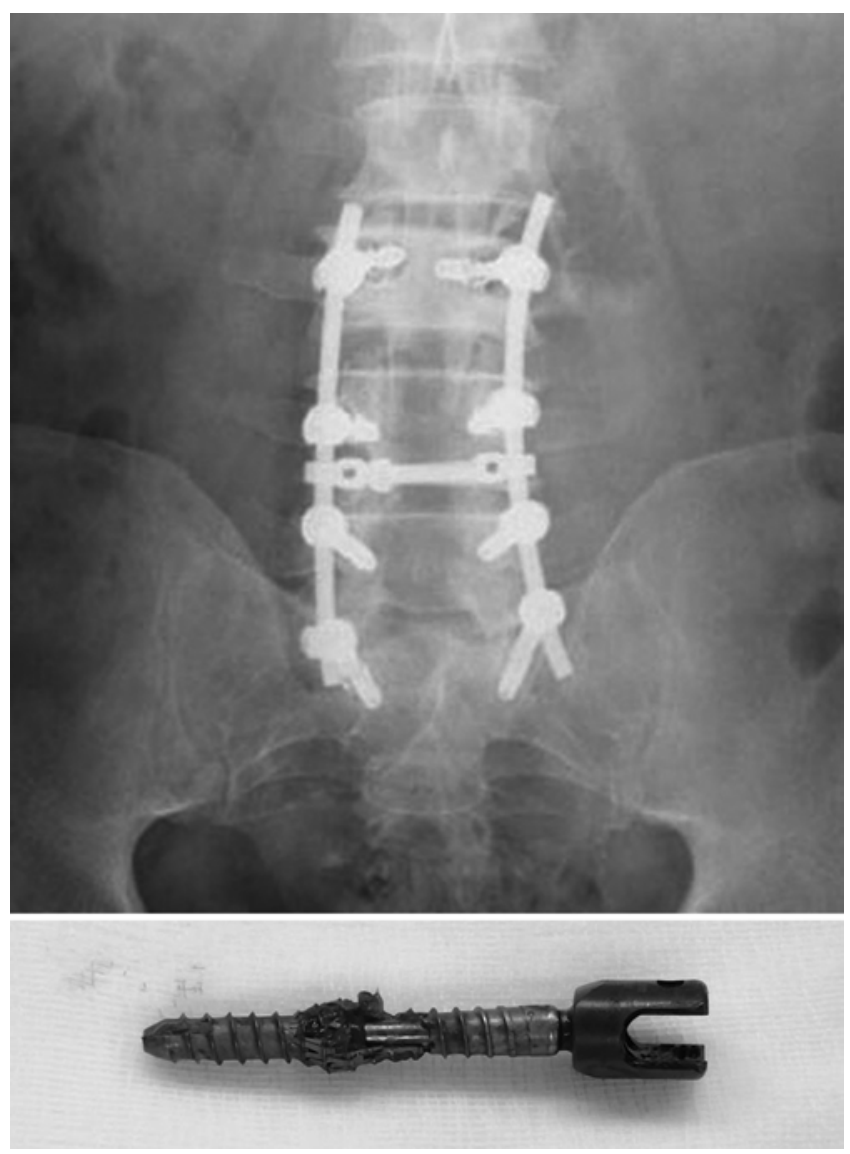

FIG. 6. Images obtained in a 61-year-old man with severe osteoporosis, who was treated for a multilevel lumbar stenosis with an L3-S1 spinal fixation. He presented at the 2-year clinical follow-up with low-back pain (VAS Score 7). Upper: Spinal radiograph at 24-month follow-up showed radiolucency around the screws at the L-3 level. Lower: Intraoperative photograph showing the L-3 screw after removal, with breakage of the outer part of the expandable screw (commercially pure titanium)

cases, the instrumentation was removed without any neurological injury. In the series reported by Cook et al. using the Omega21 spinal fixation system, the expandable screw breakage occurred in approximately $2.8 \%$ and $2.6 \%$ of the total number of expandable screws. In all cases, screw breakage occurred in the presence of pseudarthrosis. ${ }^{6,8}$ The incidence of expandable screw breakage compares favorably with that reported in the literature review by Yahiro, who reported a $7.1 \%$ rate of screw breakage for all systems. ${ }^{38}$ In our surgical series, in 1 case of 4 -segment spinal fixation (L3-S1) screw breakage occurred, with anterior migration of the expanded portion of the broken screw and loosening of the contralateral screw in the same vertebra (Fig. 6). We suggest that the long screw-rod construct was challenged by large cantilever forces acting on the endlevel anchors. The stresses imposed on the upper pedicle screw (without any anterior stabilization) may have caused screw breakage, culminating in screw migration.

In the middle of the constructs, we placed 32 expandable screws and 8 regular screws. In 1 case in which a previous kyphoplasty/vertebroplasty was performed, we used 


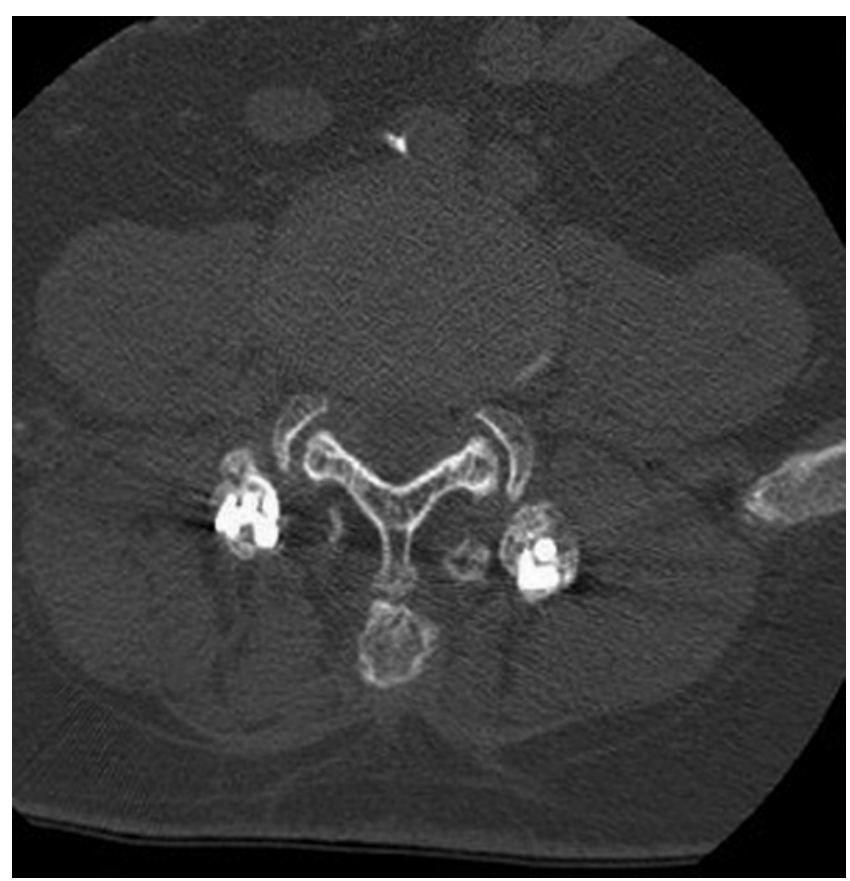

FIG. 7. Axial CT image of the spine showing fusion around the tulip of the screw.

shorter regular screws in these vertebrae and expandable screws in the upper and lower vertebrae.

Screw pullout at the proximal and distal ends of multilevel constructs represents a common spinal surgery problem, and where motion is greatest screw pullout can occur clinically more frequently than in the middle of the construct. Similarly, thoracic screws tend to pullout less than lumbar screws due to the inherent limited mobility of the thoracic spine compared with lumbar segments and thoracolumbar junction. In our series, standard screws placed in the middle of the construct had similar measurement values at follow-up as that of the expandable screws. At final radiological follow-up, in 3- and 4-segment multilevel constructs, caudal and cranial screws located in the sacrum and lumbar vertebrae showed a greater increase of screw tip distance from the anterior cortex of the vertebral body compared with thoracic screws. The migration or millimetric loosening of screws was greater at the last follow-up evaluation. In our series, only 1 case of screw failure occurred, with breakage at the distal ends of a 4-segment spinal fixation.

We never used interbody structural or fusion grafts in our patients. To visually assess the location of peak screw expansion, we used the trial probe. To achieve the desired screw depth, decortication of the facet was performed with cutting flutes located on the probe, thus promoting the fusion process around the head of the screws, providing not only a rich source of vascular supply from the underlying cancellous bone, but also accessing the pluripotent stem cells within the bone marrow. On spinal CT, we achieved fusion in 12 patients (Fig. 7).

Although OsseoScrew multiaxial expandable screws are useful for stabilizing the osteoporotic spine, they have some limitations. One limitation is the size of the screw ( $6.5 \mathrm{~mm}$ or $7.5 \mathrm{~mm}$ ); expandable screws can be used only in the sacrum and lumbar and lower thoracic spine where the pedicles are well represented. Twenty-two expandable screws were placed at L-1 and L-2 vertebrae. Pedicle size at those levels is typically smaller compared with that at other levels. To determine the appropriate screw size, the size of the pedicle was checked on the preoperative CT scan. The majority of screws used were $6.5-\mathrm{mm}$ in diameter (Fig. 8). In those few cases in which the size of the ped-
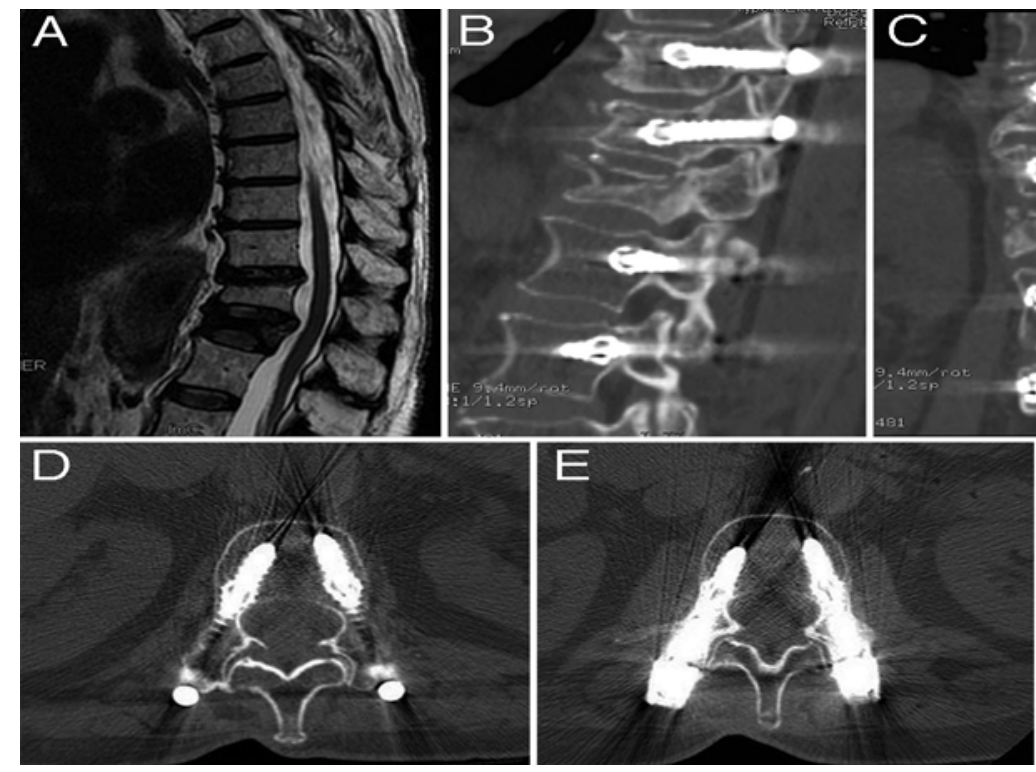

FIG. 8. This 67-year-old woman presented with an osteoporotic T-12 fracture on a sagittal MR image (A). Postoperative sagittal (B) and coronal (C) CT images show spinal fixation at T10-L2 levels with expandable pedicle screws. Axial CT images of L-1 (D) and $\mathrm{L}-2(\mathrm{E})$ demonstrate that although pedicle size at those levels is typically smaller compared with other levels, usually it is possible to use the $6.5-\mathrm{mm}$ screw after checking the pedicle size on preoperative CT scans. 
icle was too small, we used standard screws. Another limitation is screw removal. During the primary operation, the screw can be removed easily with a specific screwdriver that resizes the expansion of the screw, and the screw can then be backed out normally. During the revision surgery, bone tissue has already grown inside the expanded screw and the screw cannot return to its original shape. In our study, 2 expanded pedicle screws were removed in the same patient 24 months after surgery. The screws were extracted, removing first the inner portion and then the outer part of commercially pure titanium. The fixation system was then reinforced with a caudal and cranial fixation with expandable screws.

The achievement of a stable rigid fixation of L-5 and sacrum is a challenge to the spine surgeon. The trabecular density of the S-1 pedicle is more than that of the S-1 body. Screw fixation to the sacrum places large mechanical demands on the construct at that level. Bicortical and tricortical screw purchase and maximizing screw diameter have both been recommended to improve stability. However, bicortical and tricortical screws have the potential for neurovascular and visceral injury. In our series, pedicle screws were inserted in 9 cases at the S-1 level (Fig. 9). We used unicortical expandable screws, which may allow a greater flexibility at the S-1 level and may lower technical demands in placing sacral pedicle screws. A previous biomechanical study showed no differences between the shorter unicortical expandable screw compared with the longer bicortical standard screw. The expandable screw consistently continued to hold a high load prior to failure, resulting in a $22 \%$ increase in failure energy, compared with standard screws that exhibited a precipitous drop in load. The screw expands at the isthmus-pedicle junction, the strongest portion of the vertebral body, where cancellous bone is denser. In contrast to the regular pedicle screw that is held only within the pedicle itself (with a single-point fixation), the expandable screw has 2 points of fixation: the threads within the pedicle and the anterior pedicle/body junction. Furthermore, the expansion region of the screw compacts the dense cortical region of the pedicle, maintaining fixation. The exact location of peak expansion of the screw upon deployment is identified radiographically using a trial probe. Cutting flutes in the trial may be used to help achieve the desired screw depth and expansion zone placement.

In a biomechanical study comparing standard titanium pedicle screws and titanium expandable pedicle screws, a statistically greater amount of energy was required for the expanding screws to fail compared with the standard screws. The expanding screws consistently demonstrated a continuous level of high load as the expansion region traversed through the pedicle, while the standard screws showed a precipitous drop in load. ${ }^{33}$

Although this study has several limitations related to the relatively small patient cohort and heterogeneity of the treated pathologies, the numbers were adequate to identify implant safety and efficacy of the implant at clinical and radiological follow-up. A relative caveat of this study is also the lack of a control group of patients treated with standard titanium pedicle screws and/or with standard pedicle screws augmented with cement. One can also ar-
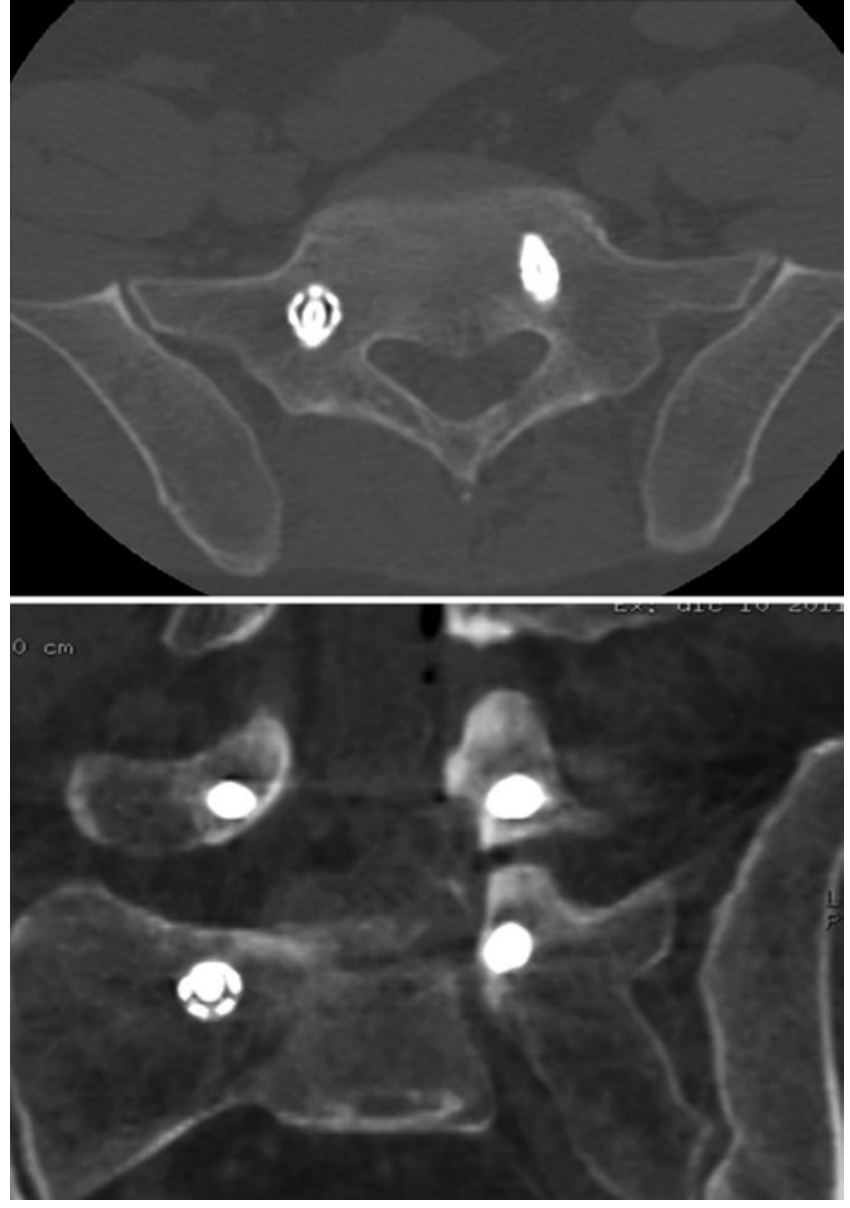

FIG. 9. Axial (upper) and coronal (lower) CT images showing screw expansion at the S-1 level.

gue whether the use of expandable screws justifies the potentially greater costs of their use. It was not the purpose of this study, however, to analyze costs. To do so, a different study protocol would be required, one reflecting a variety of complex financial and international issues, such as cost reimbursement in the different participating countries, and the need for reoperation in cases of implant failure with regular screws. Moreover, to evaluate the advantages and limitations of expandable pedicle screws, further clinical and radiological comparative studies with conventional cement augmentation screw fixation should be conducted to provide us with some guidance.

\section{Conclusions}

This study suggests that use of expandable screws is a safe, reliable technique in patients with osteoporosis who need posterior spinal fixation. OsseoScrew, thanks to its construction, allows a solid seal of the screw in the vertebral body, whereas a traditional screw does not guarantee good stability for the possible pullout of the screw itself. The material that the screw is composed of (titanium alloy and pure titanium) as well as the design allow an expansion of a region of defined geometry that 
locks the central segment of the screw into the neck of the pedicle, providing a significant stabilization in the osteoporotic spine because of its biomechanical properties. The increased fixation strength may prevent screw pullout in patients with osteoporosis and in pedicle screw revision surgery. The improvement in holding power may improve patient outcomes and possibly result in fewer implant-related complications. Further clinical and radiological studies to compare expandable screw fixation with conventional cement augmentation screw fixation should be conducted.

\section{References}

1. Becker S, Chavanne A, Spitaler R, Kropik K, Aigner N, Ogon M, et al: Assessment of different screw augmentation techniques and screw designs in osteoporotic spines. Eur Spine J 17:1462-1469, 2008

2. Bonnick SL: Osteoporosis in men and women. Clin Cornerstone 8:28-39, 2006

3. Burval DJ, McLain RF, Milks R, Inceoglu S: Primary pedicle screw augmentation in osteoporotic lumbar vertebrae: biomechanical analysis of pedicle fixation strength. Spine (Phila Pa 1976) 32:1077-1083, 2007

4. Chang MC, Liu CL, Chen TH: Polymethylmethacrylate augmentation of pedicle screw for osteoporotic spinal surgery: a novel technique. Spine (Phila Pa 1976) 33:E317-E324, 2008

5. Cho W, Cho SK, Wu C: The biomechanics of pedicle screwbased instrumentation. J Bone Joint Surg Br 92:1061-1065, 2010

6. Cook SD, Barbera J, Rubi M, Salkeld SL, Whitecloud TS III: Lumbosacral fixation using expandable pedicle screws: an alternative in reoperation and osteoporosis. Spine J 1:109-114, 2001

7. Cook SD, Salkeld SL, Stanley T, Faciane A, Miller SD: Biomechanical study of pedicle screw fixation in severely osteoporotic bone. Spine J 4:402-408, 2004

8. Cook SD, Salkeld SL, Whitecloud TS III, Barbera J: Biomechanical evaluation and preliminary clinical experience with an expansive pedicle screw design. J Spinal Disord 13:230236, 2000

9. Frankel BM, D’Agostino S, Wang C: A biomechanical cadaveric analysis of polymethylmethacrylate-augmented pedicle screw fixation. J Neurosurg Spine 7:47-53, 2007

10. Gazzeri R, Roperto R, Fiore C: Litanium expandable pedicle screw for the treatment of degenerative and traumatic spinal diseases in osteoporotic patients: preliminary experience. Surg Technol Int 22:320-325, 2012

11. Halvorson TL, Kelley LA, Thomas KA, Whitecloud TS III, Cook SD: Effects of bone mineral density on pedicle screw fixation. Spine (Phila Pa 1976) 19:2415-2420, 1994

12. Hirano T, Hasegawa K, Takahashi HE, Uchiyama S, Hara T, Washio T, et al: Structural characteristics of the pedicle and its role in screw stability. Spine (Phila Pa 1976) 22:25042510,1997

13. Hu MH, Wu HT, Chang MC, Yu WK, Wang ST, Liu CL: Polymethylmethacrylate augmentation of the pedicle screw: the cement distribution in the vertebral body. Eur Spine J 20:1281-1288, 2011

14. Kilbanski A, Adams-Campbell L, Bassford T: Osteoporosis prevention, diagnosis, and therapy. JAMA 285:785-795, 2001

15. Koller H, Zenner J, Hitzl W, Resch H, Stephan D, Augat P, et al: The impact of a distal expansion mechanism added to a standard pedicle screw on pullout resistance. A biomechanical study. Spine J 13:532-541, 2013

16. Liu D, Wu ZX, Pan XM, Fu SC, Gao MX, Shi L, et al: Biomechanical comparison of different techniques in primary spinal surgery in osteoporotic cadaveric lumbar vertebrae: expansive pedicle screw versus polymethylmethacrylateaugmented pedicle screw. Arch Orthop Trauma Surg 131:1227-1232, 2011

17. Liu D, Lei W, Wu ZX, Gao MX, Wan SY, Fu SC, et al: Augmentation of pedicle screw stability with calcium sulfate cement in osteoporotic sheep: biomechanical and screw-bone interfacial evaluation. J Spinal Disord Tech 24:235-241, 2011

18. Masaki T, Sasao Y, Miura T, Torii Y, Kojima A, Aoki H, et al: An experimental study on initial fixation strength in transpedicular screwing augmented with calcium phosphate cement. Spine (Phila Pa 1976) 34:E724-E728, 2009

19. McLain RF, McKinley TO, Yerby SA, Smith TS, SarigulKlijn N: The effect of bone quality on pedicle screw loading in axial instability. A synthetic model. Spine (Phila Pa 1976) 22:1454-1460, 1997

20. Norton RL: Machine Design: An Integrated Approach, ed 3. Upper Saddle River, NJ: Pearson Prentice Hall, 2006

21. Paré PE, Chappuis JL, Rampersaud R, Agarwala AO, Perra $\mathrm{JH}$, Erkan S, et al: Biomechanical evaluation of a novel fenestrated pedicle screw augmented with bone cement in osteoporotic spines. Spine (Phila Pa 1976) 36:E1210-E1214, 2011

22. Peebles DJ, Ellis RH, Stride SD, Simpson BR: Cardiovascular effects of methylmethacrylate cement. BMJ 1:349-351, 1972

23. Ponnusamy KE, Iyer S, Gupta G, Khanna AJ: Instrumentation of the osteoporotic spine: biomechanical and clinical considerations. Spine J 11:54-63, 2011

24. Renner SM, Lim TH, Kim WJ, Katolik L, An HS, Andersson GB: Augmentation of pedicle screw fixation strength using an injectable calcium phosphate cement as a function of injection timing and method. Spine (Phila Pa 1976) 29:E212E216, 2004

25. Röllinghoff M, Siewe J, Eysel P, Delank KS: Pulmonary cement embolism after augmentation of pedicle screws with bone cement. Acta Orthop Belg 76:269-273, 2010

26. Sarzier JS, Evans AJ, Cahill DW: Increased pedicle screw pullout strength with vertebroplasty augmentation in osteoporotic spines. J Neurosurg 96 (3 Suppl):309-312, 2002

27. Schmidt R, Cakir B, Mattes T, Wegener M, Puhl W, Richter $\mathrm{M}$ : Cement leakage during vertebroplasty: an underestimated problem? Eur Spine J 14:466-473, 2005

28. Shea TM, Laun J, Gonzalez-Blohm SA, Doulgeris JJ, Lee WE III, Aghayev K, et al: Designs and techniques that improve the pullout strength of pedicle screws in osteoporotic vertebrae: current status. BioMed Res Int 2014:748393, 2014

29. Shim CS, Lee SH, Shin HD, Kang HS, Choi WC, Jung B, et al: CHARITÉ versus ProDisc: a comparative study of a minimum 3-year follow-up. Spine (Phila Pa 1976) 32:10121018,2007

30. Skinner R, Maybee J, Transfeldt E, Venter R, Chalmers W: Experimental pullout testing and comparison of variables in transpedicular screw fixation. A biomechanical study. Spine (Phila Pa 1976) 15:195-201, 1990

31. Stadelmann VA, Bretton E, Terrier A, Procter P, Pioletti DP: Calcium phosphate cement augmentation of cancellous bone screws can compensate for the absence of cortical fixation. $\mathbf{J}$ Biomech 43:2869-2874, 2010

32. Taniwaki Y, Takemasa R, Tani T, Mizobuchi H, Yamamoto $\mathrm{H}$ : Enhancement of pedicle screw stability using calcium phosphate cement in osteoporotic vertebrae: in vivo biomechanical study. J Orthop Sci 8:408-414, 2003

33. Vishnubhotla S, McGarry WB, Mahar AT, Gelb DE: A titanium expandable pedicle screw improves initial pullout strength as compared with standard pedicle screws. Spine J 11:777-781, 2011

34. Wan S, Lei W, Wu Z, Liu D, Gao M, Fu S: Biomechanical and histological evaluation of an expandable pedicle screw in 
osteoporotic spine in sheep. Eur Spine J 19:2122-2129, 2010

35. Weinstein JN, Rydevik BL, Rauschning W: Anatomic and technical considerations of pedicle screw fixation. Clin Orthop Relat Res (284):34-46, 1992

36. Wu ZX, Gong FT, Liu L, Ma ZS, Zhang Y, Zhao X, et al: A comparative study on screw loosening in osteoporotic lumbar spine fusion between expandable and conventional pedicle screws. Arch Orthop Trauma Surg 132:471-476, 2012

37. Wu ZX, Gao MX, Sang HX, Ma ZS, Cui G, Zhang Y, et al: Surgical treatment of osteoporotic thoracolumbar compressive fractures with open vertebral cement augmentation of expandable pedicle screw fixation: a biomechanical study and a 2-year follow-up of 20 patients. J Surg Res 173:91-98, 2012

38. Yahiro MA: Comprehensive literature review. Pedicle screw fixation devices. Spine (Phila Pa 1976) 19 (20 Suppl):2274S-2278S, 1994

39. Yamamoto H, Niwa S, Hori M, Hattori T, Sawai K, Aoki S, et al: Mechanical strength of calcium phosphate cement in vivo and in vitro. Biomaterials 19:1587-1591, 1998

40. Yi X, Wang Y, Lu H, Li C, Zhu T: Augmentation of pedicle screw fixation strength using an injectable calcium sulfate ce- ment: an in vivo study. Spine (Phila Pa 1976) 33:2503-2509, 2008

\section{Disclosures}

The authors report no conflict of interest concerning the materials or methods used in this study or the findings specified in this paper.

\section{Author Contributions}

Conception and design: Gazzeri, Roperto. Acquisition of data: Gazzeri, Roperto. Analysis and interpretation of data: Gazzeri. Drafting the article: Gazzeri. Critically revising the article:

Gazzeri. Reviewed submitted version of manuscript: Gazzeri. Approved the final version of the manuscript on behalf of all authors: Gazzeri. Study supervision: Gazzeri, Fiore.

\section{Correspondence}

Roberto Gazzeri, Department of Neurosurgery, San Giovanni Addolorata Hospital, Via dell'Amba Aradam 9, Rome 00184, Italy. email: robertogazzeri@gmail.com. 